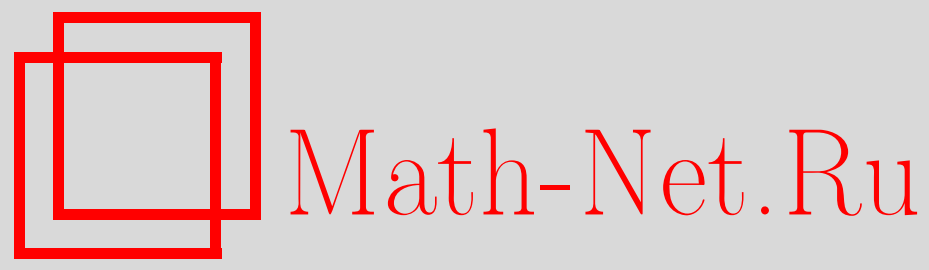

С. Л. Зиглин, Об отсутствии вещественноаналитического первого интеграла в некоторых задачах динамики, Функи. анализ и его прил., 1997, том 31, выпуск 1, 3-11

DOI: https://doi.org/10.4213/faa441

Использование Общероссийского математического портала MathNet.Ru подразумевает, что вы прочитали и согласны с пользовательским соглашением

http://www . mathnet.ru/rus/agreement

Параметры загрузки:

IP : 3.91 .87 .62

26 апреля 2023 г., 15:53:50

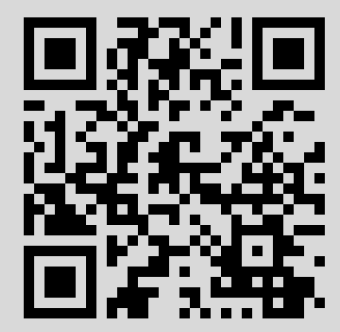


Функииональный анализ и его приложения

1997, т. 31, вып. 1, с. 3-11

УДК 517.913

\title{
Об отсутствии вещественно-аналитического первого интеграла в некоторых задачах динамики
}

\author{
(c) 1997. С. Л. Зиглин
}

1. Формулировка результатов. В $[1,2]$ было доказано отсутствие дополнительного мероморфного первого интеграла (образующего с известными функционально независимую систему) в некоторых задачах динамики в комплексифицированном фазовом пространстве.

В настоящей работе доказывается отсутствие в этих задачах мероморфного (в частности, аналитического) первого интеграла и в вещественном фазовом пространстве. Имеют место следующие теоремы.

Теорема 1. В задаче о движении тяжелого твердого тела около неподвижной точки общий дополнительный вещественно-мероморфньй ${ }^{1}$ первьй интеграл существует только в известных случалх Эйлера, Лагранжа и Ковалевской, а частный (при нулевой постоянной площадей) 一 только в трех указанньх выше случаях и в случае Горячева-Чапльгина.

ЗАмЕчАниЕ. Для динамически несимметричного тела утверждение теоремы 1 следует из $[3,4]$. Для задачи о движении динамически симметричного тяжелого твердого тела около неподвижной точки отсутствие дополнительного вещественно-аналитического первого интеграла (общего или частного) доказано в $[5,6]$ (см. также [7]) для случая, когда один из главных моментов инерции много меньше двух других (возмущенный сферический маятник), в [8, 9] (см. также $[7,10])$ для возмущенного случая Лагранжа и в [11] для случая, когда центр тяжести лежит в экваториальной плоскости и отношение главных моментов инерции больше четырех.

Теорема 2. Система Хенона-Хейлеса [12] не имеет дополнительного вешественно-мероморфного первого интеграла.

Теорема 3. В задаче Суслова [13] о движении твердого тела около неподвижной точки с неголономной связью, состоящей в том, что угловая скорость ортогональна направлению, неподвижному в системе координат, связанной с телом, в случае [14], когда указанное направление совпадает с направлением одной из главньх осей инериии в точке подвеса, тело находится в однородном поле тяжести и чентр тяжести лежит на указанной оси, дополнительный вещественно-мероморфный первый интеграл существует, только если тело динамически симметрично (аналог случая Лагранжа движения тяжелого твердого тела около неподвижной точки без дополнительных связей).

${ }^{1}$ То есть представимый в окрестности любой точки в виде отношения вещественноаналитических функций. 
2. Некоторые используемые сведения. Будем использовать следующие определения из [15]. Пусть $M$ - комплексное многообразие, $v$ - аналитическое векторное поле на $M$ и $\Gamma$ - его комплексная фазовая кривая, не являющаяся положением равновесия.

Пусть $f$ - аналитическая функция на $M$, постоянная на Г. Будем обозначать через $f^{\prime}$ функщию на нормальном расслоении $N \Gamma=T_{\Gamma} M / T \Gamma$ кривой $\Gamma$ в $M$, порожденную функцией $d f$ на $T_{\Gamma} M$. Очевидно, что $f^{\prime}$ линейна в слоях расслоения $N \Gamma$.

Пусть $H=\left(H_{1}, \ldots, H_{l}\right), l \geqslant 0$, - аналитические первые интегралы векторного поля $v$, такие, что их дифференциалы линейно независимы на Г. Будем называть приведенным фазовым пространством системы в нормальных вариациях вдоль $\Gamma$ поверхность уровня $N_{p} \Gamma=\left\{\xi \in N \Gamma \mid H^{\prime}(\xi)=p\right\}, p \in \mathbb{C}^{l}$, ее первых интегралов, а приведенной системой в вариациях ограничение этой системы на указанную поверхность.

Очевидно, что $N_{p} \Gamma$ является голоморфным аффинным (при $p=0$ векторным) расслоением над $\Gamma$, а приведенная система в вариациях линейна (при $p=0$ однородна).

Группой монодромии линейной системы в голоморфном аффинном (векторном) расслоении над римановой поверхностью будем называть образ естественного антипредставления фундаментальной группы этой поверхности в какойлибо точке в группу аффинных (линейных) преобразований слоя над этой точкой.

ПРЕДЛОЖЕНИЕ [15]. Если дифференииальное уравнение, определяемое векторным полем $v$, имеет дополнительный мероморфный первый интеграл в некоторой области $U \subset M$, такой, что фундаментальная группа фазовой кривой Г (в некоторой точке) представима петлями, лежащими в $U$, то группа монодромии приведенной системь в вариачиях вдоль Г при любом $p \in \mathbb{C}^{l}$ имеет дополнительный рациональный первый интеграл.

Будем использовать выражение «почти все» в смысле «все, кроме, может быть, счетного числа».

3. Доказательство теорем 1-3. Будем считать, что в задаче о движении тяжелого твердого тела около неподвижной точки тело является динамически симметричным. Для динамически несимметричного тела, как указано выше, результат следует из $[3,4]$.

Для каждой из рассматриваемых систем в $[1,2]$ указано инвариантное двумерное комплексное вещественно-аналитическое многообразие $\widetilde{M}^{2}$, на котором система имеет вещественно-аналитический первый интеграл (энергии) и интегрируется в квадратурах. В $[1,2]$ указано однопараметрическое семейство $\Gamma_{k}, 0<$ $k \leqslant 1$, комплексных фазовых кривых этой системы, не являющихся положениями равновесия, на которых дифференциалы известных первых интегралов $H=$ $\left(H_{1}, \ldots, H_{l}\right)$ линейно независимы, и имеет место следующее доказываемое ниже

ПреДЛОЖенИЕ 1. Для почти всех $k$ существует $p \in \mathbb{C}^{l}$, maкое, ито группа монодромии приведенной системь в вариациях вдоль фазовой кривой $\Gamma_{k}$ на приведенном фазовом пространстве $N_{p} \Gamma_{k}=\left\{\xi \in N \Gamma_{k} \mid H^{\prime}(\xi)=p\right\}^{2}$ не имеет рачионального первого интеграла.

${ }^{2}$ Строго говоря, в обозначении операции $H \rightarrow H^{\prime}$ следует указывать значение параметра $k$ кривой $\Gamma_{k}$, но, чтобы не усложнять обозначений, условимся этого не делать. 
Из предложения 1 и предложения, сформулированного в разд. 2, вытекает

ПРЕДЛОЖЕНИЕ 2. Ни одна из рассматриваемых систем не имеет дополнительного мероморфного первого интеграла ни в какой области $U$ комплексифичированного фазового пространства, такой, что для более чем счетного числа значений $k$ фундаментальная группа фазовой кривой $\Gamma_{k}$ представима петлями, лежащими в $U$.

ДОКАЗАТЕЛЬСТВо ПРЕДЛОЖЕНИЯ 1. Покажем, что для всех рассматриваемых систем, кроме задачи о движении тяжелого твердого тела около неподвижной точки в случае Горячева-Чаплыгина, предложение 1 справедливо уже при $p=0$.

Действительно, для системы Хенона-Хейлеса в [1] доказано, что искомый первый интеграл может существовать только при значениях параметра $k$, при которых собственные значения некоторого двумерного линейного симплектического преобразования, аналитически зависящего от $k$, не равные тождественно $\pm i$, равны $\pm i$. Очевидно, что число таких значений не более чем счетно.

Для задачи о движении тяжелого твердого тела около неподвижной точки в случае, когда центр тяжести не лежит в экваториальной плоскости, в [1] доказано, что указанный первый интеграл не может существовать на всем интервале $0<k<1$, однако доказательство справедливо и для любого подмножества этого интервала, имеющего предельную точку, в частности, для любого более чем счетного подмножества.

Для задачи о движении тяжелого твердого тела около неподвижной точки в случае, когда центр тяжести лежит в экваториальной плоскости, и для задачи Суслова из $[1,2]$ следует, что:

(I) Приведенная система в вариациях инвариантна относительно некоторого инволютивного диффеоморфизма, не имеющего неподвижных точек и линейного в слоях приведенного фазового пространства (порожденного инволютивным диффеоморфизмом исходного фазового пространства, явно выписанным в $[1,2])$.

(II) Группа монодромии факторсистемы этой системы по действию указанного диффеоморфизма может иметь рациональный первый интеграл только при значениях параметра $k$, при которых собственные значения некоторого двумерного линейного симплектического преобразования, аналитически зависящего от $k$, не равные тождественно корням из единицы, равны корням из единицы. Ясно, что число таких значений не более чем счетно.

Так как очевидно, что линейная система в голоморфном векторном расслоении над римановой поверхностью имеет мероморфный первый интеграл тогда и только тогда, когда ее группа монодромии имеет рациональный первый интеграл, то из (II) следует, что

(III) При почти всех $k$ указанная факторсистема не имеет мероморфного первого интеграла.

Из (I), (III), согласно лемме п. 1.5 из [15], следует, что при этих значениях $k$ приведенная система в вариациях также не имеет мероморфного первого интеграла, а отсюда, в свою очередь, следует, что ее группа монодромии не имеет рационального первого интеграла, что и требовалось доказать.

Остается доказать предложение 1 для случая Горячева-Чаплыгина. В этом случае система Эйлера-Пуассона, описывающая движение тяжелого твердого 
тела около неподвижной точки, при соответствующем выборе единиц измерения и направлений главных осей инерции в точке подвеса [1] имеет вид

$$
\begin{gathered}
\dot{M}_{1}=3 M_{2} M_{3}, \quad \dot{M}_{2}=-3 M_{1} M_{3}+\gamma_{3}, \quad \dot{M}_{3}=-\gamma_{2}, \\
\dot{\gamma}_{1}=4 M_{3} \gamma_{2}-M_{2} \gamma_{3}, \quad \dot{\gamma}_{2}=-4 M_{3} \gamma_{1}+M_{1} \gamma_{3}, \quad \dot{\gamma}_{3}=M_{2} \gamma_{1}-M_{1} \gamma_{2} .
\end{gathered}
$$

Здесь $\vec{M}=\left(M_{1}, M_{2}, M_{3}\right), \vec{\gamma}=\left(\gamma_{1}, \gamma_{2}, \gamma_{3}\right)$ - кинетический момент тела и единичный вектор, направленный против силы тяжести, в проекциях на главные оси инерции в точке подвеса.

Будем рассматривать систему (1) в комплексифицированном фазовом пространстве $M^{5}=\left\{x=(\vec{M}, \vec{\gamma}) \in \mathbb{C}^{6} \mid \vec{\gamma}^{2}=1\right\}$. Она имеет функционально независимые «общие» первые интегралы:

$$
H=\frac{1}{2}\left(M_{1}^{2}+M_{2}^{2}\right)+2 M_{3}^{2}+\gamma_{1}
$$

(энергии) и

$$
P=\langle\vec{M}, \vec{\gamma}\rangle
$$

(площадей), а на многообразии $M_{0}^{4}=\left\{x \in M^{5} \mid P(x)=0\right\}$ - дополнительный «частный» первый интеграл

$$
F=M_{3}\left(M_{1}^{2}+M_{2}^{2}\right)-M_{1} \gamma_{3}
$$

Система (1) имеет инвариантное двумерное многообразие $\widetilde{M^{2}} \subset M_{0}^{4}$, задаваемое в $M^{5}$ уравнениями $M_{1}=M_{3}=\gamma_{2}=0$.

На многообразии $\widetilde{M^{2}}$ она имеет однопараметрическое семейство решений $x=$ $\tilde{x}(t, k), 0<k \leqslant 1$, задаваемое формулами

$$
\begin{gathered}
M_{1}=M_{3}=\gamma_{2}=0, \quad M_{2}=\widetilde{M}_{2}(t, k)=-2 k \operatorname{cn}(t, k), \\
\gamma_{1}=\tilde{\gamma}_{1}(t, k)=2 k^{2} \operatorname{sn}^{2}(t, k)-1, \quad \gamma_{3}=\tilde{\gamma}_{3}(t, k)=2 k \operatorname{sn}(t, k) \operatorname{dn}(t, k), \\
k=\sqrt{(1+h) / 2},
\end{gathered}
$$

где $\operatorname{sn}(t, k), \operatorname{cn}(t, k), \operatorname{dn}(t, k)$ - эллиптические функции Якоби с модулем $k$, a $h$ - постоянная интеграла энергии.

Эти решения однозначны, мероморфны, а при $0<k<1$, что далее и будет предполагаться, и двоякопериодичны по $t$ с периодами $T_{1,2}(k)=2 K(k) \pm$ $2 i K^{\prime}(k)$, где $K(k)$ — полный эллиптический интеграл первого рода с модулем $k, K^{\prime}(k)=K\left(k^{\prime}\right), k^{\prime}=\sqrt{1-k^{2}}$, и имеют в ячейках по два полюса $a_{1,2}(k)= \pm i K^{\prime}(k)\left(\bmod T_{1,2}(k)\right)$. Обозначим их фазовые кривые через $\Gamma_{k} \cdot$

Приведенная система в вариациях вдоль фазовой кривой $\Gamma_{k}$ на приведенном фазовом пространстве $N_{p} \Gamma_{k}=\left\{\xi \in N \Gamma_{k} \mid H^{\prime}(\xi)=0, P^{\prime}(\xi)=p\right\}, p \in \mathbb{C}$, имеет вид

$$
\begin{gathered}
\dot{M}_{1}^{\prime}=3 \widetilde{M_{2}}(t, k) M_{3}^{\prime}, \quad \dot{M}_{3}^{\prime}=-\gamma_{2}^{\prime}, \\
\dot{\gamma}_{2}^{\prime}=\tilde{\gamma}_{3}(t, k) M_{1}^{\prime}-4 \tilde{\gamma}_{1}(t, k) M_{3}^{\prime}, \quad \tilde{\gamma}_{1}(t, k) M_{1}^{\prime}+\tilde{\gamma}_{3}(t, k) M_{3}^{\prime}+\widetilde{M}_{2}(t, k) \gamma_{2}^{\prime}=p .
\end{gathered}
$$


Пусть $\alpha_{i, k}:[0,1] \rightarrow \Gamma_{k}(i=1,2)$ - гладкие петли с общей отмеченной точкой, такие, что их поднятия $\beta_{i, k}:[0,1] \rightarrow \mathbb{C}$ относительно $\tilde{x}(\cdot, k)$ суть соответственно прямолинейный путь, такой, что $\beta_{1, k}(1)-\beta_{1, k}(0)=4 K(k)$, и петля, однократно обходящая полюс $a_{1}(k)$ решения $x=\tilde{x}(t, k)$ в положительном направлении, и пусть $g_{i, k, p}$ - соответствующие им преобразования монодромии.

Будем называть аффинное преобразование жордановым, если оно имеет неподвижную точку и матрица его линейной части является жордановой клеткой.

Собственными векторами и собственными значениями аффинного преобразования будем называть собственные векторы и собственные значения его линейной части.

Обозначим через $F_{k, p}^{\prime}$ ограничение функции

$$
F^{\prime}=\widetilde{M}_{2}^{2}(t, k) M_{3}^{\prime}-\tilde{\gamma}_{3}(t, k) M_{1}^{\prime}: N \Gamma_{k} \rightarrow \mathbb{C}
$$

на слой приведенного фазового пространства $N_{p} \Gamma_{k}$ над точкой $\alpha_{i, k}(0)$.

Лемма. Преобразование $g_{1, k, p}$ при почти всех $k$ и всех $p$ является жордановым с единичныл собственным значением и собственным вектором, обращаюшим в нуль 1-форму $F_{k, 0}^{\prime}$, a преобразование $g_{2, k, p}$ при всех $k u$ $p \neq 0$ - сдвигом на вектор, не обращаюший в нуль эту 1-форму ( $а$ при $p=0$ - тождественньм преобразованием).

Из этой леммы следует (cp. $[16,17])$ отсутствие рационального первого интеграла группы монодромии при почти всех $k$ и всех $p \neq 0$. Действительно, он должен быть постоянным как на всех прямых, параллельных собственному вектору преобразования $g_{1, k, p}$, так и на всех прямых, параллельных вектору сдвига преобразования $g_{2, k}$, , и, следовательно, тождественно постоянным, что и требовалось доказать.

ДокАЗАтЕЛЬСтво ЛЕммы. (А) Случай $p=0$. При $p=0$ система (3) имеет первый интеграл $F^{\prime}$ и интегрируется в квадратурах. Ее общее решение имеет вид

$$
\begin{gathered}
M_{1}^{\prime}=\widetilde{M}_{2}^{3}(t, k)(c+3 f J(t, k)), \\
M_{3}^{\prime}=\widetilde{M}_{2}(t, k) \tilde{\gamma}_{3}(t, k)(c+3 f J(t, k))+f \widetilde{M}_{2}^{-2}(t, k), \\
\gamma_{2}^{\prime}=-\left(\widetilde{M}_{2}^{2}(t, k) \tilde{\gamma}_{1}(t, k)+\widetilde{\gamma}_{3}^{2}(t, k)\right)(c+3 f J(t, k))-f \widetilde{M}_{2}^{-3}(t, k) \tilde{\gamma}_{3}(t, k), \\
J(t, k)=\int_{t_{0}(k)}^{t} \widetilde{M}_{2}^{-4}(\tau, k) d \tau .
\end{gathered}
$$

Здесь $c, f$ - произвольные постоянные, причем $f$ - постоянная интеграла $F^{\prime}, t_{0}:(0,1) \rightarrow \mathbb{C}$ - произвольная функция, такая, что $t_{0}(k) \neq b_{m, n}(k)$, где $b_{m, n}(k)=n K(k)+i(m+2 n-1) K^{\prime}(k)(m, n$ целые $)$ - нули и полюсы функции $M_{2}=\widetilde{M}_{2}(t, k)$; интеграл берется по любому кусочно-гладкому пути, не проходящему через точки $b_{m, n}(k)$.

Из формул (4) следует, что:

(I) Это решение однозначно, и, следовательно, $g_{2, k, 0}=\mathrm{id}$. Действительно, так как матрица правой части системы (3) имеет особенности только в полюсах решения $x=\tilde{x}(t, k)$, то ее решение (4) могло бы иметь ветвление только в этих 
точках, но, как следует из (2), в этих точках подынтегральное выражение в интеграле $J(t, k)$ регулярно, и, следовательно, этот интеграл, а значит, и все решение (4) однозначны.

(II) При $f=0$ это решение имеет период $4 K(k)$ и, следовательно, преобразование $g_{1, k, 0}$ имеет неподвижный вектор.

Для доказательства леммы в случае $p=0$ остается проверить, что при $f \neq 0$ и почти всех $k$ число $4 K(k)$ не является периодом этого решения, и, следовательно, преобразование $g_{1, k, 0}$ жорданово.

Пусть $\beta_{k}:[0,1] \rightarrow \mathbb{C}$ - прямолинейный путь, такой, что $\beta_{k}(1)-\beta_{k}(0)=$ $4 K(k), \operatorname{Im} \beta_{k}(0) \neq n K^{\prime}(k), n$ целое. Положим

$$
\Delta(k)=J\left(\beta_{k}(1), k\right)-J\left(\beta_{k}(0), k\right) .
$$

Имеем

$$
\Delta(k)=\int_{\beta_{k}} \widetilde{M}_{2}^{-4}(t, k) d t .
$$

Переходя к новой переменной интегрирования $\theta$ по формулам $\tilde{\gamma}_{1}(t, k)=$ $-\sin \theta, \tilde{\gamma}_{3}(t, k)=\cos \theta(\theta$ при $0 \leqslant \theta<\pi$ совпадает с эйлеровым углом нутации), получаем

$$
\Delta(k)=2^{-5 / 2} \int_{\delta_{k}}(h+\sin \theta)^{-5 / 2} d \theta, \quad h=2 k^{2}-1,
$$

где $\delta_{k}:[0,1] \rightarrow \mathbf{S}_{\mathbb{C}}^{1}$ - петля на комплексифицированной окружности $\mathbf{S}_{\mathbb{C}}^{1}=$ $\mathbb{C} / 2 \pi \mathbb{Z}$ с угловой координатой $\theta(\bmod 2 \pi)$, однократно обходящая отрезок $I_{k}=$ $[-\arcsin h, \pi+\arcsin h]$. Направление обхода и ветвь подынтегрального выражения связаны следующим образом: при стягивании петли $\delta_{k}$ к отрезку $I_{k}$ вещественная часть подынтегрального выражения положительна на той стороне отрезка, на которой движение вдоль петли происходит в направлении увеличения $\operatorname{Re} \theta$.

Отсюда получаем

$$
\begin{aligned}
\Delta(k) & =\frac{1}{3 \sqrt{2}} \frac{d^{2}}{d h^{2}} \int_{\delta_{k}}(h+\sin \theta)^{-1 / 2} d \theta \\
& =\frac{2 \sqrt{2}}{3} \frac{d^{2}}{d h^{2}} \int_{-\arcsin h}^{\pi / 2}(h+\sin \theta)^{-1 / 2} d \theta=\frac{4}{3} \frac{d^{2}}{d h^{2}} K(\sqrt{(1+h) / 2}) .
\end{aligned}
$$

Так как $K(k) \rightarrow \infty$ при $k \rightarrow 1$, то $\Delta(k) \neq 0$ при почти всех $h$, а значит, и при почти всех $k$, что и требовалось доказать.

(Б) Случай $p \neq 0$. Поскольку при любых $i, k, p$ линейная часть преобразования $g_{i, k, p}$ совпадает с $g_{i, k, 0}$, для доказательства леммы остается проверить, что

(i) при почти всех $k$ и всех $p$ преобразование $g_{1, k, p}$ сохраняет линейную Функцию $F_{k, p}^{\prime}$;

(ii) при всех $k$ и $p \neq 0$ преобразование $g_{2, k, p}$ не сохраняет эту функцию.

Действительно, из (A) и (i) следует, что при почти всех $k$ и всех $p$ преобразование $g_{1, k, p}$ имеет жорданову линейную часть и сохраняет прямые, параллельные ее собственному вектору. Отсюда следует, что оно имеет неподвижную точку и, значит, само является жордановым. 
Из (А) и (ii) следует, что преобразование $g_{2, k, p}$ при всех $k$ и $p \neq 0$ является сдвигом на вектор, не обращающий в нуль 1 -форму $F_{k, 0}^{\prime}$. Утверждение доказано.

Обозначим через $f_{p}$ функцию на фазовой кривой $\Gamma_{k}$, такую, что $f_{p}(x)$ равно значению производной $\dot{F}^{\prime}$ функции $F^{\prime}$ в силу систем $(1),(3)$ в слое приведенного фазового пространства $N_{p} \Gamma_{k}$ над точкой $x$ (оно постоянно в этом слое, так как Функция $\dot{F}^{\prime}$ линейна в слоях нормального расслоения $N \Gamma_{k}$ и равна нулю на приведенном фазовом пространстве $N_{0} \Gamma_{k}$ ).

Полагая

$$
\Delta_{i}(k, p)=F_{k, p}^{\prime} \circ g_{i, k, p}-F_{k, p}^{\prime}, \quad i=1,2,
$$

имеем

$$
\Delta_{i}(k, p)=\int_{\beta_{i, k}} f_{p}(\tilde{x}(t, k)) d t .
$$

Из (1), (3), (2) получаем

$$
f_{p}(\tilde{x}(t, k))=-p \widetilde{M}_{2}(t, k)=2 p \operatorname{cn}(t, k),
$$

откуда $\Delta_{1}(k, p)=0$,

$$
\Delta_{2}(k, p)=4 p k \pi i \operatorname{Res} \operatorname{cn}\left(i K^{\prime}(k), k\right)=4 \pi p \neq 0
$$

при $p \neq 0$. Лемма и предложение 1 доказаны.

Каждая из рассматриваемых систем имеет на многообразии $\widetilde{M}^{2}$ вещественную неподвижную гиперболическую точку $x_{0}$. Вещественная часть фазовой кривой $\Gamma_{1}$ содержит компоненты, являющиеся вещественными фазовыми кривыми решений, стремящихся к $x_{0}$ при $t \rightarrow \pm \infty$. (Для системы Хенона-Хейлеса такая компонента одна, а для остальных рассматриваемых систем таких компонент две, и их объединение совпадает с $\operatorname{Re} \Gamma_{1}$.) Обозначим через $\Omega$ замыкание их объединения.

ПРЕДЛОЖЕНИЕ 3. Для любой комплексной окрестности $U \subset \widetilde{M^{2}}$ множества $\Omega$ существует $\varepsilon>0$, такое, что при $0<|k-1|<\varepsilon$ фундаментальная группа фазовой кривой $\Gamma_{k}$ представима петлями, лежащими в $U$.

ЗАмЕЧАниЕ. Предложение 3 справедливо и при $k=1$, однако для дальнейшего это не требуется.

Из предложений 2 и 3 следует отсутствие у рассматриваемых систем дополнительного мероморфного первого интеграла в любой комплексной, а значит, и в вещественной области фазового пространства, содержащей $\Omega$, что и доказывает теоремы 1-3.

ДоКАЗАТЕЛЬСТво ПРЕДЛОЖЕНИя 3. Для каждой из рассматриваемых систем в $[1,2]$ указано однопараметрическое семейство решений $x=\varphi(t, k)$, соответствующих фазовым кривым $\Gamma_{k}$. При $0<k<1$ эти решения однозначны, мероморфны, двоякопериодичны по $t$ и вещественны при вещественных значениях $t$.

Пусть $T(k)$ и $T^{\prime}(k)$ - минимальные вещественный и чисто мнимый периоды решения $x=\varphi(t, k)$. Для системы Хенона-Хейлеса они являются примитивными (т.е. любой период является их целочисленной линейной комбина- 
цией), и решение имеет в ячейках по одному полюсу $T^{\prime}(k) / 2\left(\bmod T(k), T^{\prime}(k)\right)$, a для остальных рассматриваемых систем примитивными являются периоды $T_{1,2}(k)=\left(T(k) \pm T^{\prime}(k)\right) / 2$, и решение имеет в ячейках по два полюса $\pm T^{\prime}(k) / 4$ $\left(\bmod T_{1,2}(k)\right)$.

Положим для системы Хенона-Хейлеса $t_{0}(k)=T(k) / 2$, а для остальных рассматриваемых систем $t_{0}(k)=T(k) / 4$. Пусть $\gamma_{k}, \gamma_{k}^{\prime}:[0,1] \rightarrow \Gamma_{k}-$ петли с общей отмеченной точкой $x_{k}=\varphi\left(t_{0}(k), k\right)$, соответствующие изменению времени вдоль периодов $T(k), T^{\prime}(k)$ решения $x=\varphi(t, k)$. Из сказанного выше следует, что для системы Хенона-Хейлеса гомотопические классы петель $\gamma_{k}, \gamma_{k}^{\prime}$ являются образующими фундаментальной группы $\pi_{1}\left(\Gamma_{k}\right)$, а для остальных рассматриваемых систем эти петли пересекаются еще в одной точке $x_{k}^{\prime}=\varphi\left(t_{0}(k)+T(k) / 2, k\right)=\varphi\left(t_{0}(k)+T^{\prime}(k) / 2, k\right)$ и фундаментальная группа $\pi_{1}\left(\Gamma_{k}\right)$ представима петлями, состоящими из полупетель этих петель с концами в точках $x_{k}, x_{k}^{\prime}$.

Из формул, приведенных в $[1,2]$, следует, что при $k \rightarrow 1$ точка $x_{k}$ стремится к $x_{0}$, петля $\gamma_{k}$ - к множеству $\Omega$, а петля $\gamma_{k}^{\prime}-$ к точке $x_{0}$ (последнее следует также из нормальной формы рассматриваемых систем на многообразии $\widetilde{M}^{2}$ в окрестности точки $x_{0}$ или из непрерывной зависимости решения дифференциального уравнения от начального условия и ограниченности периода $T^{\prime}(k)$ на любом интервале $0<k_{0}<k<1$ ). Предложение 3 доказано.

Автор благодарен В. И. Арнольду, А. Д. Брюно, В. В. Козлову, А. И. Нейштадту и А. И. Овсеевичу за полезные обсуждения.

Пользуясь случаем, исправим следующие неточности, замеченные в работах $[1,15]$.

В [1] в первом слагаемом в подынтегральном выражении в (13) должно быть $\sin 2\left(\chi_{0}+\omega x\right)$, а не $\sin \left(2 \chi_{0}+\omega x\right)$.

В уравнении $\chi^{1}\left(\pi q, \chi_{0}\right)=0$ после формулы (19) выражение в скобках должно быть равно $-1 / 4+\omega^{2}$, а не $-1 / 4+1 / \omega^{2}$, и, следовательно, обращается в нуль при $\omega=1 / 2$, а не при $\omega=2$.

Дополнительный первый интеграл для случая Горячева-Чаплыгина должен иметь вид $F=M_{3}\left(M_{1}^{2}+M_{2}^{2}\right)-M_{1} \gamma_{3}$, как в настоящей работе, а не $F=$ $M_{3}\left(M_{1}^{2}+M\right)_{2}^{2}-M_{1} \gamma_{3}$.

Приведенное в [1] доказательство отсутствия частного дополнительного мероморфного первого интеграла в задаче о движении тяжелого твердого тела около неподвижной точки во всех случаях, кроме известных случаев интегрируемости, является вследствие предложения п. 1.2 из [15] (предложение разд. 2 настоящей работы) одновременно доказательством отсутствия в этих случаях общего дополнительного мероморфного первого интеграла, так что отдельное доказательство этого утверждения, приведенное в [1], так же как и сформулированное для него замечание к теореме В. В. Козлова в [15], могут быть опущены.

В алгебраической лемме п. 1.2 из [15] пропущено условие соизмеримости весов $s_{1}, \ldots, s_{m}$ (т.е. должно быть $s_{i}=n_{i} s, n_{i} \in \mathbb{Z}, i=1, \ldots, m, s \in \mathbb{R}$ ), нужное для того, чтобы описанный в доказательстве леммы процесс понижения показателя зависимости $\mu\left(\Phi_{1}, \ldots, \Phi_{r}\right)$ исходной системы функций $\Phi_{1}, \ldots, \Phi_{r}$ был конечен. 
В то же время условие неотрицательности весов $s_{1}, \ldots, s_{m}$ в случае поля рациональных функций излишне и в доказательстве не используется. Вследствие этого алгебраическая лемма в [18] также становится излишней.

\section{ЛИТЕРАТУРА}

1. Зиглин C. Л. Ветвление решений и несуществование первых интегралов в гамильтоновой механике, II. Функц. анализ и его прил., 17, вып. 1, 8-23 (1983).

2. Зиглин $C$. Л. Об отсутствии дополнительного первого интеграла в одной задаче динамики твердого тела. ДАН СССР, 292, №4, 804-807 (1987).

3. Зизлин C. Л. Расщепление сепаратрис, ветвление решений и несуществование интеграла в динамике твердого тела. Труды МMO, 41, 287-303 (1980).

4. Зиглин C. Л. Расщепление сепаратрис и несуществование первых интегралов в системах дифференциальных уравнений типа гамильтоновых с двумя степенями свободы. Изв. АН СССР, сер. матем., 51, №5, 1088-1103 (1987).

5. Козлов B. B., Трещев Д. В. Неинтегрируемость общей задачи о вращении динамически симметричного тяжелого твердого тела с неподвижной точкой, I. Вестник МГУ, сер. матем., мех., №6, 73-81 (1985).

6. Козлов B. В., Трещев Д. В. Неинтегрируемость общей задачи о вращении динамически симметричного тяжелого твердого тела с неподвижной точкой, II. Вестник МГУ, сер. матем., мех., №1, 39-44 (1986).

7. Козлов B. B. Симметрии, топология и резонансы в гамильтоновой механике. Изд-во Удмуртского гос. ун-та, Ижевск, 1995.

8. Болотин $C$. B. Двоякоасимптотические траектории и условия интегрируемости гамильтоновых систем. Вестник МГУ, сер. матем., мех., №1, 55-63 (1990).

9. Довбыш C. A. Расщепление сепаратрис неустойчивых равномерных вращений и неинтегрируемость возмущенной задачи Лагранжа. Вестник МГУ, сер. матем., мех, №3, 70-77 (1990).

10. Довбыш C. A. О сепаратрисе неустойчивого положения равновесия волчка Гесса-Аппельрота. ПММ, 56, вып. 4, 632-642 (1992).

11. Болотин C. В. Вариационные методы построения хаотических движений в динамике твердого тела. ПММ, 56, вып. 2, 230-239 (1992).

12. Henon $M$., Heiles $C$. The applicability of the third integral of motion; some numerical experiments. Astron. J., 69, 73-79 (1964).

13. Суслов Г. К. Теоретическая механика. Гостехиздат, М.-Л., 1946.

14. Козлов B. B. К теории интегрирования уравнений неголономной механики. Успехи механики, 8, вып. 3, 85-107 (1985).

15. Зиглин $C$. Л. Ветвление решений и несуществование первых интегралов в гамильтоновой механике, I. Функц. анализ и его прил., 16, вып. 3, 30-41 (1982).

16. Козлов $B$. В. Несуществование однозначных интегралов и ветвление решений в динамике твердого тела. ПММ, 42, вып. 3, 400-406 (1978).

17. Козлов B. B. Ветвление решений и полиномиальные интегралы в обратимой системе на торе. Матем. заметки, 44, вып. 1, 100-104 (1988).

18. Зиглин C. Л. О полиномиальных первых интегралах гамильтоновых систем с экспоненциальным взаимодействием. Функц. анализ и его прил., 25, вып. 3, 88-89 (1991). 